\title{
Multifunctional coatings of exfoliated and reassembled graphite on cellulosic substrates $\uparrow$
}

\author{
Leandra Pereira Santos, (D) *ad Douglas Soares da Silva, (ID) a \\ João Paulo Ferreira Bertacchi, ${ }^{a}$ Kelly Schneider Moreira, (D) b \\ Thiago Augusto Lima Burgo, (D) b Bruno Carreira Batista, (D) \\ Jeferson dos Santos, ${ }^{a}$ Patrick Alvarenga de Paula ${ }^{a}$ \\ and Fernando Galembeck (D) *ad
}

\author{
Received 23rd October 2019, Accepted 7th January 2020 \\ DOI: $10.1039 / c 9 f d 00109 c$
}

\begin{abstract}
Exfoliated and reassembled graphite (ERG) forms macroscopic, high aspect ratio $\left(1:>10^{6}\right)$ and highly conductive coating layers that are strongly adherent to paper, wood, cloth, ceramic and other substrates. The coating precursor is an aqueous dispersion of graphite that exfoliates spontaneously in alkaline cellulose solutions, forming stable dispersions. These can be applied to the substrates by using different painting, coating and lithography techniques. The coating morphology changes from highly smooth to porous and rough, depending on the finishing procedure used. Coated paper sheets are flexible and they perform as leads in electrical circuitry and as electrodes in electrodeposition, supercapacitors, hygroelectricity cells and other electrochemical devices suitable for flexible and wearable electronics. These unique properties of ERG are explained as a consequence of the amphiphilic character of cellulose, which allows it to play the roles of exfoliant, dispersant, stabilizer, adhesive and plasticizer, while graphite powder is transformed into a cohesive laminated nanocomposite.
\end{abstract}

\section{Introduction}

The outstanding properties of graphene and related 2D materials (GRM) have been attracting great attention and they are being explored to create nanomaterials that perform multiple functions in various applications. ${ }^{1-13}$ Composites are the most common industrial graphene-based products, but the quality of

\footnotetext{
${ }^{a}$ Institute of Chemistry, University of Campinas, Campinas, 13083-970, SP, Brazil.E-mail: fernagal@unicamp. br; leandrapereiradossantos@gmail.com

${ }^{b}$ Physics and Chemistry Department, Univ. Fed. Santa Maria, Santa Maria, RS, Brazil 'Institute of Chemistry, Univ. Fed. do Mato Grosso do Sul, Campo Grande, MS, Brazil ${ }^{d} G G$ \& FG Consulting \& Development, Campinas, 13084-723, SP, Brazil

$\dagger$ Electronic supplementary information (ESI) available. See DOI: 10.1039/c9fd00109c
} 
commercially available graphene is usually low compared to the materials obtained in the laboratory.

Graphene is often commercially supplied as a powder and it is not expected that $<1 \mathrm{~nm}$ thin, electroneutral sheets will remain well separated when they touch each other, due to the omnipresent van der Waals forces.

Thus, single-layer graphene cannot exist as a dry powder under normal terrestrial conditions. This is one of the factors preventing widespread use of graphene in the many industrial applications where it could be the basis for much radical innovation.

Spontaneous graphene aggregation is accompanied by significant changes in its physical properties. For instance, the electrical conductivity of graphene is among the highest known, surpassing copper and matching silver. However, piling up graphene sheets lowers its conductivity steeply: the electrical conductivity of a 100-layer lamella is in the same range as that of graphite. On the other hand, the resistance of a single graphene layer is $33 \mathrm{ohms}$ per square, too high for any applications requiring high currents. Based on data for single-wall carbon nanotubes,${ }^{14}$ graphene's ability to sustain a high current may be as high as the $10^{12} \mathrm{~A} \mathrm{~m}^{-2}$ range, thus a 1 metre wide sheet could hold a $50 \mathrm{~A}$ current. Different approaches are thus being taken to explore graphene in high-ampacity ${ }^{15}$ materials for power transmission, as well as in thermal ${ }^{16}$ or strengthening ${ }^{17,18}$ applications through the formation of graphene composites, e.g. the superhelix graphene/ copper nanocomposite wires ${ }^{19}$ with $600 \mu \mathrm{m}$ overall diameter which can carry $5.8 \times 10^{10} \mathrm{~A} \mathrm{~m}^{-2}$.

Moulding powders facilitates particle aggregation, so that we cannot think of moulded graphene macroscopic monoliths. Graphite itself can be shaped in macroscopic bars, cylinders and plates, allowing its use in power applications, but this is in turn limited by the brittleness of graphite monoliths.

In this laboratory, we approached this problem by exfoliating graphite in aqueous alkaline cellulose solutions, thus producing dispersions suitable for making conductive coatings or monoliths. ${ }^{20}$ Graphite exfoliation has been described in the literature by using various dispersing agents and by oxidizing to graphene oxide. Direct exfoliation in water is achieved using dispersant solvents, surfactants and polymers, high pressure, ${ }^{21}$ extended sonication ${ }^{22}$ and mechanochemical action (e.g. in the presence of melamine), ${ }^{23}$ followed by freeze-drying. This introduces new problems due to the negative effect of exfoliant residues which are often electrical insulators. Graphite exfoliation by cellulose takes place at dispersant/graphite weight ratios lower than 1:60; the dispersant cellulose becomes an excellent adhesive when the medium $\mathrm{pH}$ is lowered.$^{24}$ Wet cellulose is a low- $T_{\mathrm{g}}$ polymer that acts as a plasticizer of the exfoliated lamellae. Most importantly, the thin exfoliated lamellae reassemble on the substrates, forming extended, continuous and insoluble films. This combination of properties is not matched by any other dispersant, notwithstanding all the efforts reported in the literature.

Graphite exfoliation and reassembly can then produce macroscopic micronthick layers of a new material, extending over many square meters and perhaps much greater areas. The aspect ratio (width/thickness) of this material is $10^{6}$ or higher, well above the aspect ratio of a single graphene sheet with 10 microns width, and thus it performs as a macroscopic 2D material. This paper describes 
different applications of exfoliated and reassembled graphite (ERG) that may contribute to increasing the strategic and economic impact of 2D materials.

\section{Results and discussion}

Graphite exfoliated in aqueous cellulosic solutions forms non-Newtonian fluids, appearing as liquid dispersions, pastes or self-standing gels. When sheared, they show rheo-optical effects, as expected for reflective particles with large aspect ratios. The dispersions may be stored and used for many months without significant change in their performance except for an increase in viscosity due to spontaneous further exfoliation. They wet and spread on various types of lignocellulosic or ceramic surfaces: wood, paper, cardboard, cloth, charcoal, brick, china and a ceramic fibre mat. Detailed information on the dispersion properties is beyond the scope of this paper but will be published in the near future.

\section{Substrate coating and finishing}

Different coating techniques, like spraying, spreading, painting with roll or brush, bathing, silk-screening and lithography, have already been used to produce uniform and adhesive coatings with thickness ranging from some nanometres to higher than a hundred microns, on smooth or rough surfaces that may have complex shapes. Some examples are pictured in the ESI (Fig. S1†).

Successive coating layers may be applied to the substrate, allowing water to evaporate in between successive applications. Thus, it is possible to repair damaged coatings or to use additive manufacture techniques with these materials.

Upon drying, the exfoliated graphite lamellae reassemble, forming a continuous adherent film, irrespective of the substrate roughness. Drying under air is concurrent with $\mathrm{CO}_{2}$ uptake which lowers the $\mathrm{pH}$ and renders cellulose insoluble. The amphiphilic character of cellulose allows its adhesion to different lignocellulosic and ceramic materials. Cellulose is thus a good binding agent for making compatible multiphasic solid coatings.

The ERG surface is a rough network of cellulose fibrils spread over flat areas, as shown in the optical micrograph in Fig. 1. The intensity ratios of Raman spectra acquired from different spots show the presence of graphite and other kinds of graphene assemblies ${ }^{25}$ while the intensity of the cellulose fluorescence band is highly variable.

The ERG coatings may be finished by various techniques to produce smooth surfaces, as shown in Fig. 2.

The SEM micrograph of the finished ERG surface shows a flat layer of irregular particles that are predominantly aligned along the surface plane. Cellulose is also seen in the coating side view as a material with irregular shape, lying on the lamellae flat surfaces and also in between them. Cellulose occupies only a fraction of the conductive graphitic particle surfaces, allowing for electrical contact across the whole coating layer.

AFM micrographs in various modes (topography, friction force and conductive probe) are shown in Fig. 3, and they provide valuable information on ERG assembly. The topography measurements show plates with thickness of a few tens of nanometers as well as steps that are a few nm thick. 

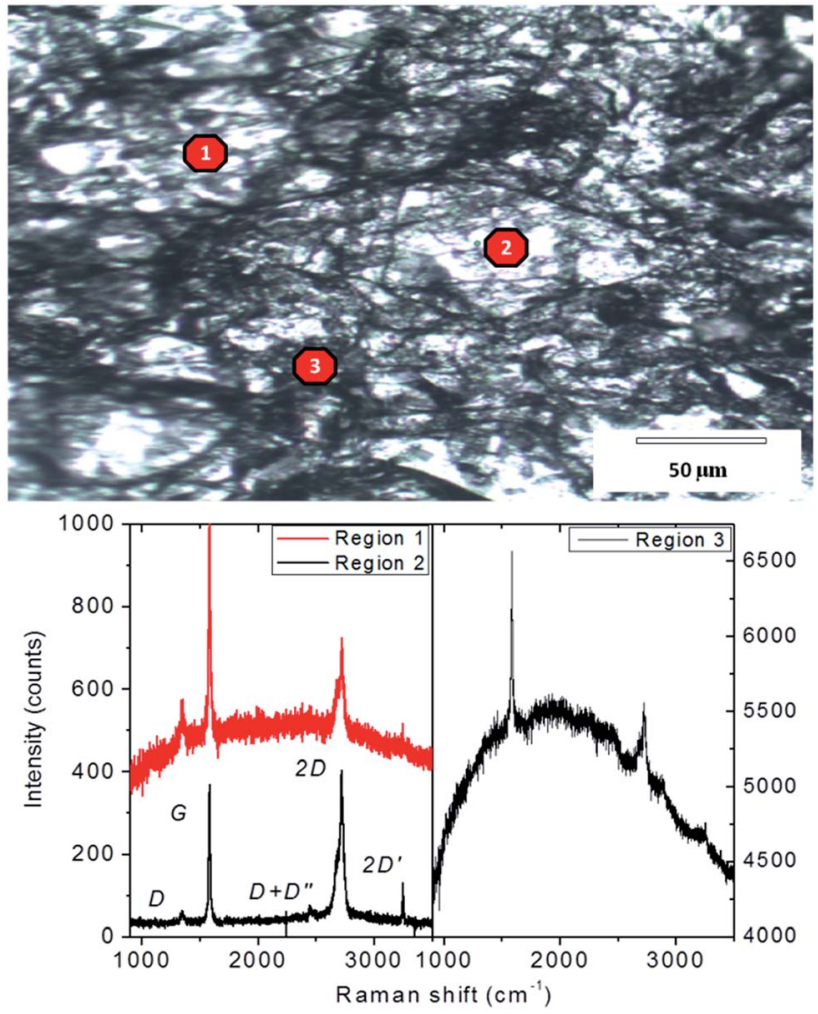

Fig. 1 Top: Optical micrograph of ERG-coated paper surface. Bottom: Raman spectra obtained from 3 different points. The Raman laser spot diameter is ca. 1 micron.

The friction force micrographs show that the surface has extensive low friction domains, interspersed with higher friction areas, while the conductive probe images verify the coexistence of conductive and non-conductive domains.

The coatings are thus anisotropic macroscopic sheets made by joining anisotropic particles aligned along the surface plane. Different from common graphite materials, ERG layers are flexible, and they can be made stiffer or softer by reversibly plasticizing the interspersed cellulose with water vapor or liquid.

Coated substrates can be bent to low curvature radii, and mechanical damage due to bending is easily repaired by retouching, or by self-repair triggered by wetting and pressing of the defective area. Coating plasticity is assigned to the unprecedented ERG microstructure shown in the micrographs, in which wide bare lamellar surfaces are held together by strong cellulose fibrils and adhesive layers that occupy only a fraction of their surface area, leaving most conductive surfaces free to contact their neighbours.

\section{Electrical resistance at the macro and nanoscales}

The low surface resistance of ERG-coated paper has already been described in previous work, but significant improvement has been achieved since the original publication. Exploring various graphite and cellulose raw materials over a broad range of compositions and using various finishing procedures provided a number 

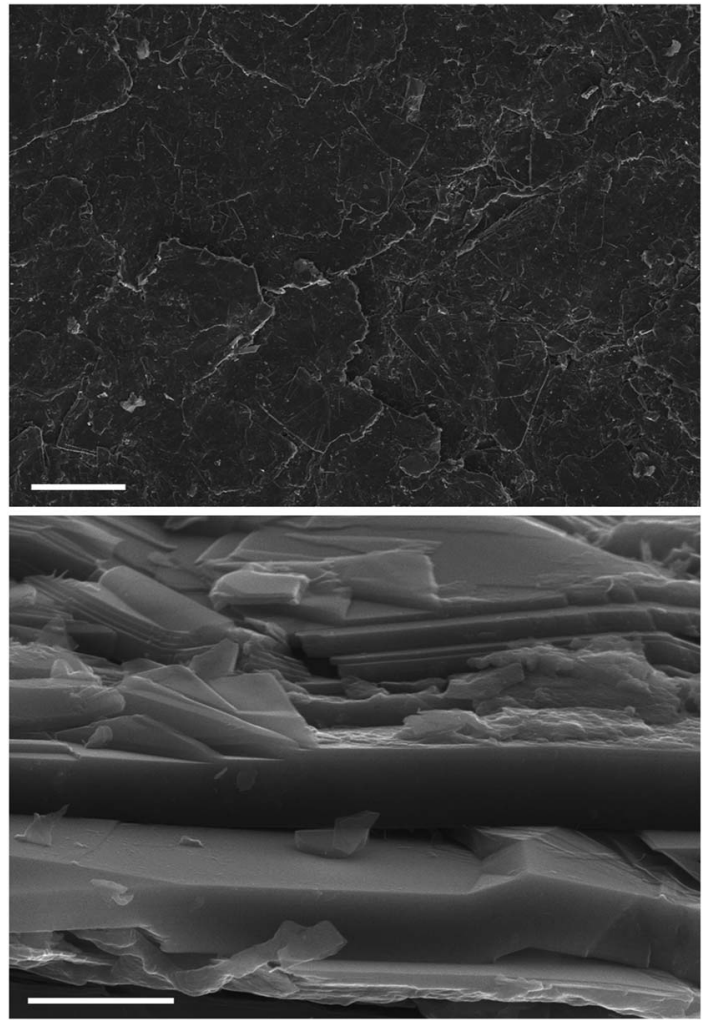

Fig. 2 Top: SEM micrograph of an ERG layer painted on paper. Bottom: View of a cut side surface. Scale bars: top, $100 \mu \mathrm{m}$; bottom, $2 \mu \mathrm{m}$.

of formulations suitable for specific purposes. As expected, dispersions with higher graphite/cellulose weight ratios produced coatings with lower surface resistance.

Fig. 4 shows resistance measurements taken along a $1 \mathrm{~cm}$ wide strip of an ERG-coated paper sheet, both as a recently prepared dry film and after calendering. The coating thicknesses were respectively 124 and $83 \mu \mathrm{m}$. From the slopes and intercepts of these plots, we obtain 22.4 and $1.1 \mathrm{ohms}$ per square for the surface resistance and 10.9 and $2.1 \mathrm{ohms}$ for the contact resistance of the unfinished and finished paper, respectively. Combined with the coating thicknesses, this yields $2.8 \times 10^{-3}$ and $9.1 \times 10^{-5}$ ohms per $\mathrm{m}$ for the resistivity, respectively.

These figures may be too high or low for some desired applications, but they are easily changed by changing the composition of the dispersion used, the sample thickness, the finishing procedure and the contact with the metal probes. This can be better understood by looking at the SEM images (Fig. 2) and especially the scanning probe micrographs (Fig. 3d), in which electrical current passes predominantly through the graphite sheets, but decreases abruptly in the cellulose-rich regions. 

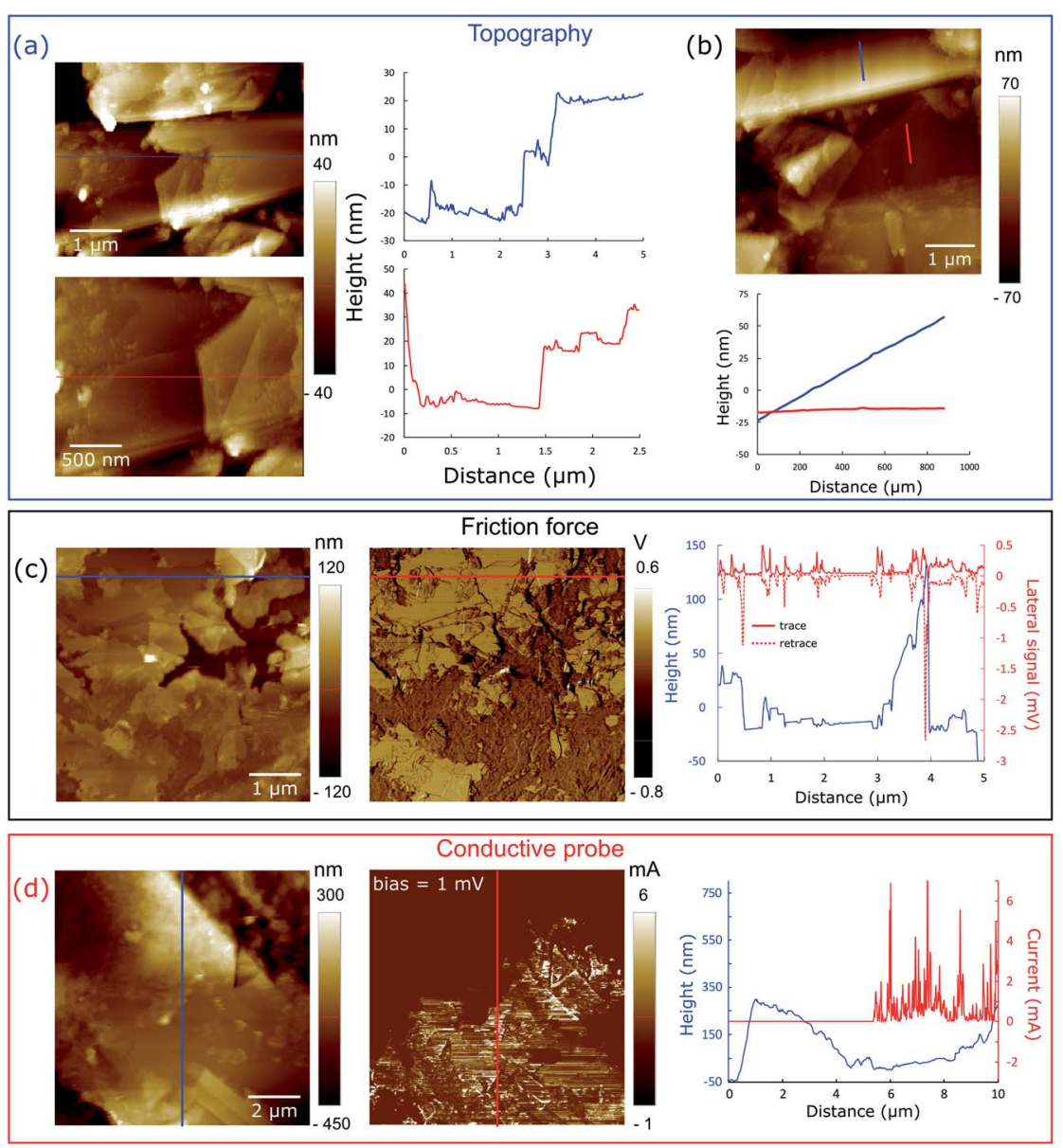

Fig. 3 Scanning probe microscopy images of a calendered ERG coating on paper. (a) and (b) Topography images and line-scans showing the morphological aspects of the nanocomposite. (c) Friction force microscopy showing height and lateral force plots along the line-scans drawn over the micrographs. (d) Conductive probe AFM: topography (left) and current (right) mode images of the calendered surface of ERG-coated paper. Height and current plots along the vertical lines drawn in the images show that the graphite domains exhibit a high electric current response.

So far, the lowest surface resistance achieved in this laboratory is $0.6 \mathrm{ohms}$ per square for another film of ERG-coated, washed and calendered Kraft paper, followed by $0.7 \mathrm{ohms}$ per square for a $40 \times 40 \mathrm{~cm}$ ERG-coated medium density fiberboard (MDF).

Further resistance lowering is probably still possible, by improving the juxtaposition of the lamellae and by reducing the amount of cellulose in the finished coating.

\section{Coating adhesion}

ERG coatings do not peel off from flexed coated paper, cardboard and cloth. Sharp bends may fracture thicker coatings, but these also resist peeling, even adjacent to 


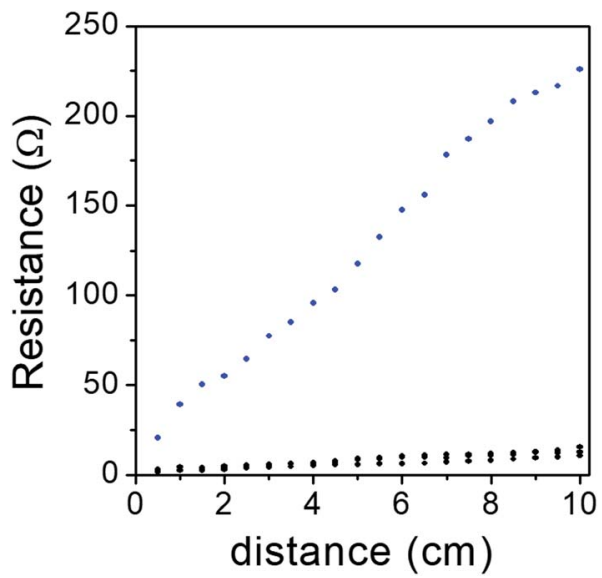

Fig. 4 Electrical resistance of $1 \mathrm{~cm}$ wide as-prepared (blue dots) ERG-coated paper sheets and calendered (black dots) ERG-coated paper sheets, as a function of distance between the measuring probe tips.

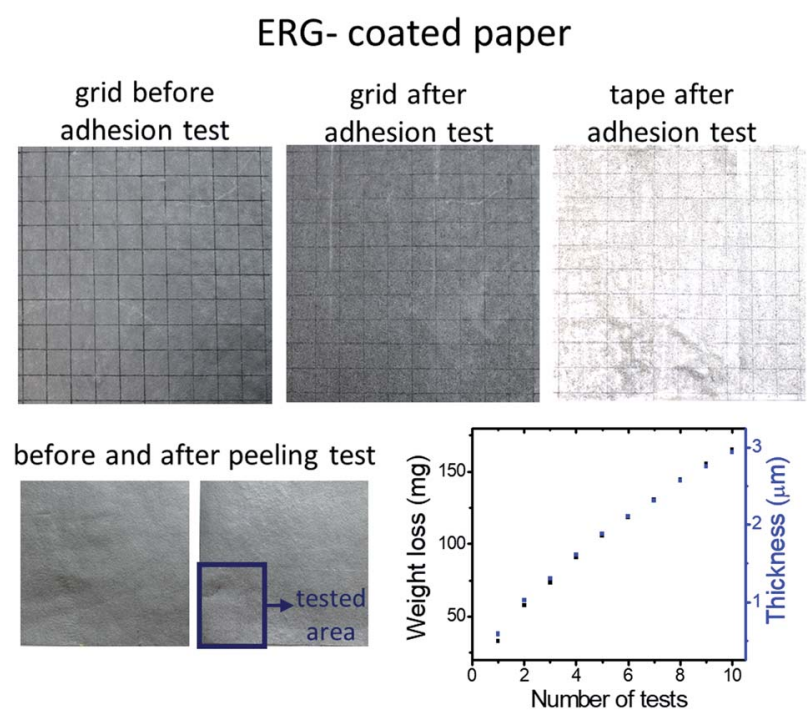

tapes after $\mathrm{N}$ tests to the same area

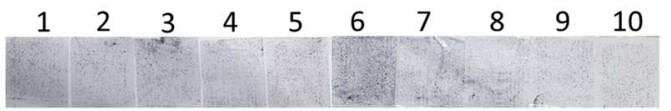

Fig. 5 Top row: Picture of a piece of ERG-coated paper that was tested for coating adhesion (left), the ERG-coated paper after the adhesion test (center), and the adhesive tape used in the test (right). Center row: A sheet of another sample, where the lower left corner layers within the lamellae, together with a small amount of adhesive cellulose that persists within the coating, were successfully coated with adhesive tape and peeled off ten times (left, center), and a plot of sample weight loss and the corresponding decrease in thickness, calculated using the weight data (right). Bottom: Pictures of the ten tapes used. 
the fracture line. ERG coating adhesion was tested following the ASTM D 3359-97 Standard, and the results pictured in Fig. 5 show that no square in the testing grid was withdrawn, and neither were the grid square tips damaged, as shown in Fig. 5, top row. The left side shows a picture of the grid before the adhesion test, and the picture in the center shows the grid after the adhesive tape was peeled off and does not show visible damage. The light discoloration of the tape used in this test is due to a small amount of delaminated graphene lamellae, evidencing that adhesion between the piled-up graphene layers is much lower than ERG adhesion to the substrate. The amount of peeled off ERG was quantified as shown in the center row in Fig. 5, showing that ten successive peeling tests removed only a small amount of the surface coating material. This is visualized in the bottom row, confirming that the successive peeling steps removed approximately the same amounts of graphene/nanographite, as shown in the weight loss plot. Together, these results show that ERG coatings may be used to prevent unwanted adhesion on the coated surfaces, since the adherent bodies can be easily peeled off, as the adhesive tapes were in this experiment.

Thus, regenerated graphite coatings display an unusual combination of adhesive properties: they are strongly adherent to the substrate, but not to external adherent bodies.

This is expected, considering the poor adhesion among graphene layers.

\section{Wetting and swelling}

The wetting behavior of the graphite coatings depends largely on their specific composition and finish, as shown by the contact angle measurements presented in Fig. 6. As-prepared dry ERG-coated paper is wetted by water and the testing droplet is slowly drawn to the underlying paper sheet. This is drastically changed by calendering, which increases the initial water-ERG contact angle, keeping it nearly steady during droplet evaporation. Coating with polydimethylsiloxane increases the contact angle, which levels off at just below $90^{\circ}$.

The remarkable effect of calendering on water contact angle is expected, considering the decrease in surface roughness accompanying the realignment of the surface lamellae under pressure. This also explains the negligible change in contact angle during droplet evaporation, considering that advancing and receding contact angles are expected to be identical on smooth, chemically uniform surfaces.

\section{Conductive leads in electric circuits}

Conductive inks ${ }^{26}$ are often designed to make printed and flexible circuitry. ERG coatings can be used in this way, and ERG paper is easily cut, coated and assembled as the leads in circuit boards. This has been demonstrated by building kits for students experimenting on electricity and electrochemistry.

Fig. 7 shows a simple circuit for distinguishing conductive from nonconductive materials, assembled from a kit that includes parts used in eleven electrochemical experiments.

\section{Electrodes}

ERG-coated paper, wood, brick and other materials are robust electrodes for various applications. For instance, copper electrodeposition is easily done, 

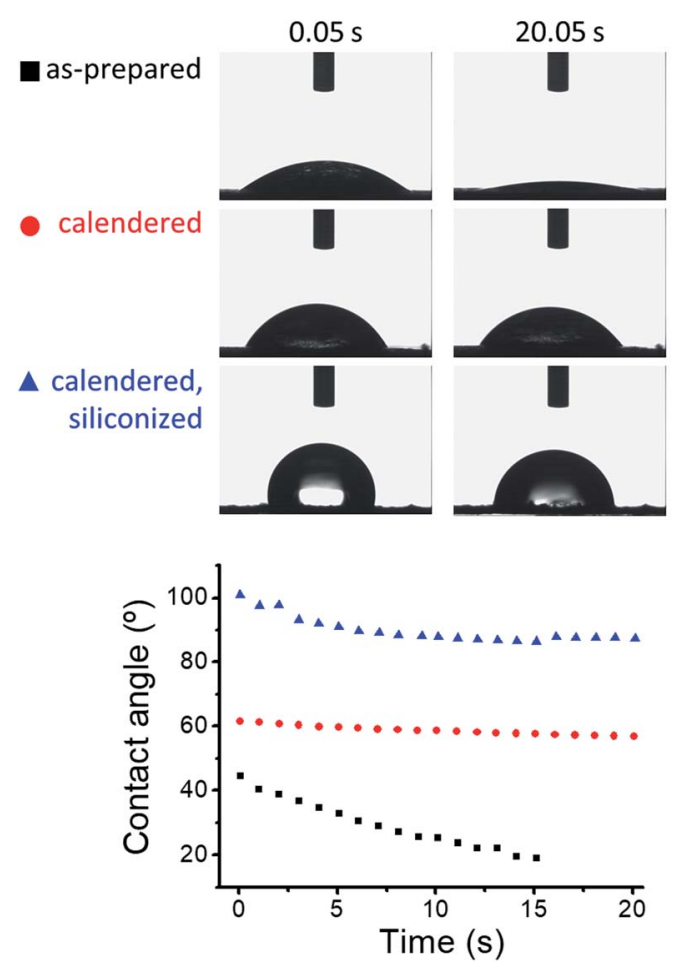

Fig. 6 Advancing contact angle measurements of $5 \mu \mathrm{L}$ sessile water drops resting on samples of paper coated with ERG.
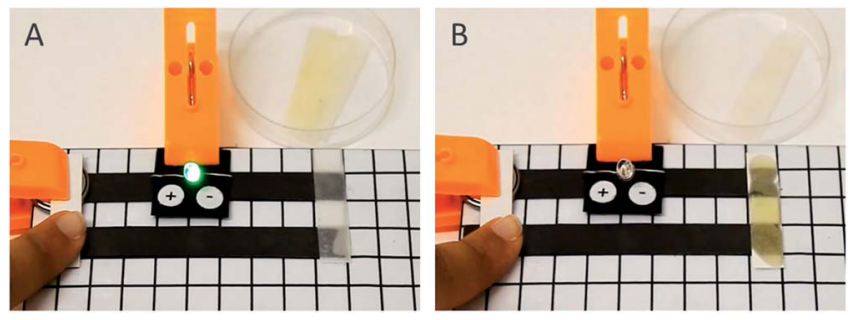

Fig. 7 Pictures of a circuit board assembled from a kit made for student experiments on electricity and electrochemistry. A piece of filter paper wetted with $(A)$ an aqueous solution of $\mathrm{NaCl}\left(0.1 \mathrm{~mol} \mathrm{~L}^{-1}\right)$ and $(B)$ cooking oil is placed between the conductive leads. A LED indicates the onset of electric current and it is lit on the left only.

producing copper films on the coated paper sheets, as shown in Fig. 8. Surface coverage by deposited copper follows the expected linear relationship with $t^{1 / 2}$.

\section{Energy applications}

Graphite and graphene perform multiple roles in electrochemical systems, especially supercapacitors ${ }^{27,28}$ and cells or batteries. 

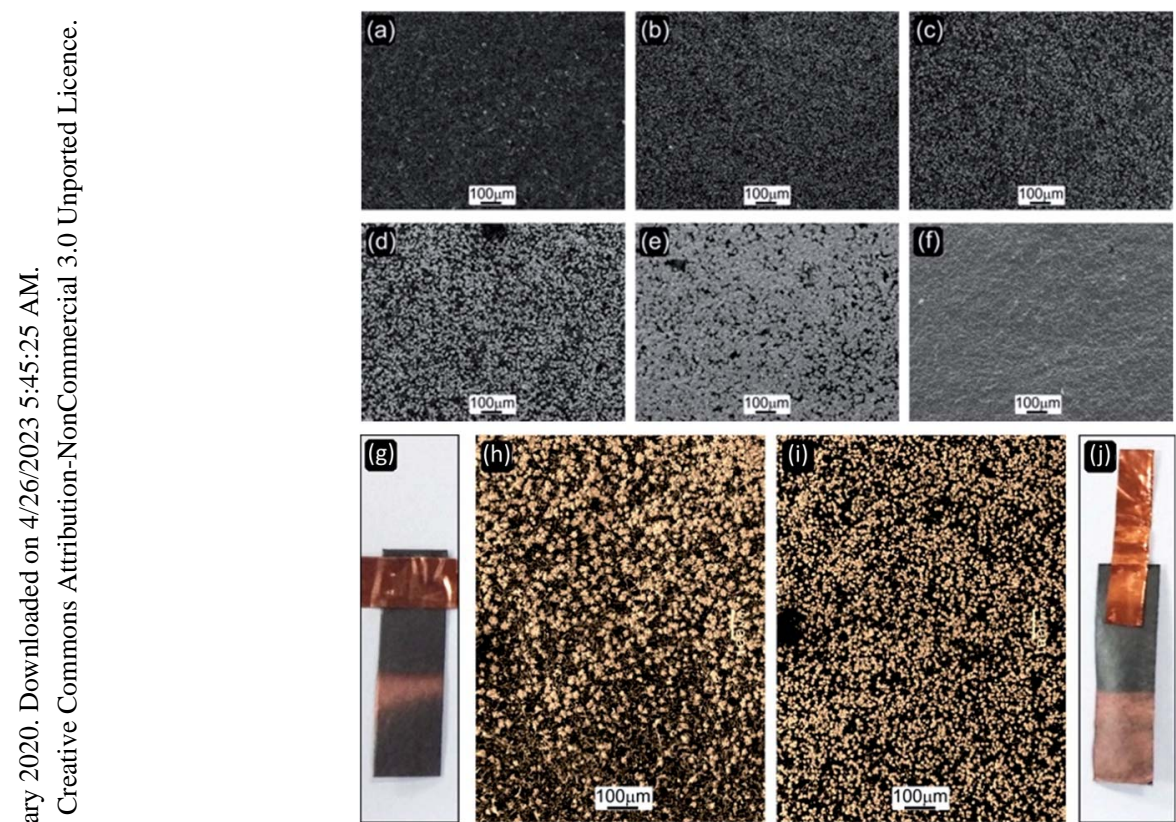

Fig. 8 Upper: SEM micrographs of copper electrodeposited on an ERG-coated calendered surface, using a $0.5 \mathrm{~mol} \mathrm{~L}^{-1}$ solution at $2.85 \mathrm{~mA} \mathrm{~cm}^{-2}$. (a) is the initial sample and (b) -(f) are samples withdrawn from the copper solution after 5, 10, 30, 60 and 120 minutes, respectively. Lower: Two ERG-coated paper strips ( $g$ and j) used for copper electrodeposition are shown together with optical micrographs ( $h$ and i) of the copper coated areas. The sample on the right was calendered, the other was not.

Electric double-layer capacitors. Electrodes for supercapacitors and other related devices show better performance when they have a large surface area and are easily penetrated by the electrolyte. For this reason, ERG was modified by adding activated charcoal to the dispersions used to make supercapacitor

Table 1 Selected data for supercapacitors made from flexible electrodes and showing high performance ${ }^{29}$

\begin{tabular}{|c|c|}
\hline Components & $\begin{array}{l}\text { Capacitance } \\
\left(\mathrm{mF} \mathrm{cm}^{-2}\right)\end{array}$ \\
\hline $\mathrm{Ni}_{3} \mathrm{~V}_{2} \mathrm{O}_{8} @ \mathrm{PANI}^{30}$ & 585 \\
\hline Annealed carbon cloth $^{31}$ & 2900 \\
\hline $\mathrm{Ni}(\mathrm{OH})_{2} / \mathrm{C} / \mathrm{Cu} / / \mathrm{Mn}_{3} \mathrm{O}_{4} / \mathrm{C} / \mathrm{Cu}^{32}$ & 216 \\
\hline ERG on paper (this work) & 350 \\
\hline Activated carbon (AC) on polyimide film ${ }^{33}$ & 35 \\
\hline Graphene/AC on polyimide film ${ }^{19}$ & 37 \\
\hline Graphene on polyimide film ${ }^{19}$ & 8 \\
\hline $\mathrm{MnCo}_{2} \mathrm{O}_{4}$ hollow spheres on carbon fibres ${ }^{34}$ & 800 \\
\hline P3MT/HACNT on PDMS ${ }^{35}$ & 3100 \\
\hline HACNT on PDMS ${ }^{21}$ & 750 \\
\hline Buckypaper on PDMS ${ }^{21}$ & 250 \\
\hline Graphene-graphite/PU ${ }^{36}$ & 16 \\
\hline
\end{tabular}


electrodes. This material showed relatively low capacitance per unit weight, but its capacitance per unit area compares favourably to other materials, as seen in Table 1.

This can be understood by considering the possibility of making thick ERG coatings, so that loading the electrode with electroactive material compensates for its low specific capacitance.

Using higher amounts of electrode materials is facilitated by their low cost, great availability and the possibility of designing and building capacitors as parts of manufactured goods, housing and equipment. This can be done without any safety concerns, since EDL capacitor voltages seldom exceed 2.5 volts.

Desired combinations of electrode properties are achieved by adding other components to ERG and using suitable processes and finishing techniques to tailor the electrodes to different applications. It is thus possible to combine the conductive properties of ERG not only with activated charcoal but also with many other components, to achieve the capacitance or pseudo capacitance shown e.g. by nickel vanadate@PANI, $\mathrm{MnCo}_{2} \mathrm{O}_{4}$ hollow spheres, poly(3-methylthiophene)/ horizontally aligned carbon nanotubes (P3MT/HACNT) on PDMS, or iron hydroxide nanoparticles which were recently disclosed as a new benchmark.

Hygroelectricity ${ }^{37-47}$ is a recent development for energy scavenging, based on water ion partition during adsorption-desorption in a moist atmosphere or pure water. It has been implemented in different ways, and a simple experimental setup is an asymmetric capacitor under humid air or wetted by pure water. It can be observed by assembling capacitors using two paper electrodes coated with

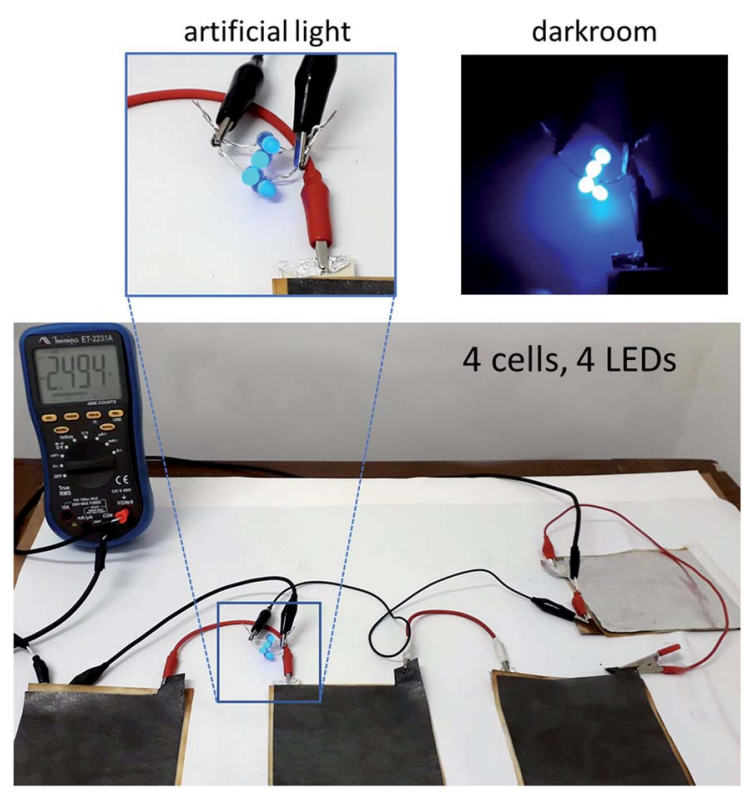

Fig. 9 Electric circuit consisting of four hygroelectricity cells in series with four blue LEDs. The hygroelectricity cell is formed by an aluminum electrode and an ERG-coated paper electrode. A piece of water-soaked filter paper placed between the electrodes keeps the cell humid. The closed-circuit voltage is approximately $2.5 \mathrm{~V}$. 
different graphite dispersions, or a capacitor built using a conductive paper electrode and an aluminium sheet.

The power output of a series arrangement is sufficient for energizing lowpower applications, fully independent of the grid and without consuming valuable metal electrodes from electrochemical cells and batteries. A simple setup that keeps many LEDs continuously lit for a few days is shown in Fig. 9.

\section{Friction, fire resistant surfaces and electrical heaters}

Beyond its electrical properties, graphite is used as a lubricant and it is resistant to fire. For this reason, ERG coatings show useful anti-friction and fire resistance properties. Beyond that, its tunable electrical resistance makes it suitable to be used in low-power density heaters. Heating uses a significant part of the energy consumed globally and it is often done using heating elements operating at much higher temperature than the heated bodies, decreasing the heater efficiency.

\section{Benchmarking}

The procedures used to obtain the oriented graphite coatings and the results achieved differ from current graphene and graphite-based inks or paints, ${ }^{7}$ opening many possibilities for the development of new products designed for different applications.

A comparison of ERG with current conductive inks and coatings and some reference materials is shown in Table 2. Coating resistance is often expressed as

Table 2 Resistivity and surface resistance of ERG, compared to related or competing materials

\begin{tabular}{|c|c|c|c|}
\hline Material & $\begin{array}{l}\text { Resistivity } \\
\left(\Omega \mathrm{m}^{-1}\right)\end{array}$ & $\begin{array}{l}\text { Resistance } \\
(\Omega \text { per square })\end{array}$ & $\begin{array}{l}\text { Thickness } \\
(\mathrm{m})\end{array}$ \\
\hline Graphene monolayer & $10^{-8}$ & 33 & $\begin{array}{l}3 \times 10^{-10}(\text { single } \\
\text { sheet })\end{array}$ \\
\hline Multilayer graphene ${ }^{49}$ & $10^{-6}$ & 33 & $\begin{array}{l}3 \times 10^{-8}(100 \\
\text { sheets })\end{array}$ \\
\hline $\begin{array}{l}\text { Graphene thin films exfoliated under high } \\
\text { pressure }^{21}\end{array}$ & $2 \times 10^{-5}$ & & 3 to $50 \times 10^{-6}$ \\
\hline $\begin{array}{l}\text { Graphene thin films exfoliated under } \\
\text { extended sonication }{ }^{22}\end{array}$ & $2.3 \times 10^{-3}$ & & \\
\hline Graphite (basal) & $2 \times 10^{-6}$ & 0.3 & $\begin{array}{l}6 \times 10^{-6}(20 \mathrm{k} \\
\text { sheets })\end{array}$ \\
\hline $\begin{array}{l}\text { ERG (including substrate, best result in this } \\
\text { lab) }\end{array}$ & & 0.6 & \\
\hline Microfluidized graphite ${ }^{50}$ & $2.5 \times 10^{-4}$ & 2 & \\
\hline Commercial silver ink ${ }^{51}$ & $\begin{array}{l}12.5 \times \\
10^{-5}\end{array}$ & $5 \times 10^{-3}$ & $2.5 \times 10^{-6}$ \\
\hline SWNT dispersed in SDBS ${ }^{52}$ & & 4 & \\
\hline $\mathrm{MnO}_{2}$-coated hollow carbon microspheres ${ }^{53}$ & & 3 & \\
\hline CNTs coated active carbon ${ }^{54}$ & & 20 & \\
\hline Silver nanowires ${ }^{55}$ & & 8 & \\
\hline $\begin{array}{l}\text { Encapsulated graphene/CuNT/EVA/PET } \\
\text { hybrid films } 56\end{array}$ & & 8 & \\
\hline
\end{tabular}


the resistance measured by contacting the surface with the probe leads and it is expressed in ohms per square, $R=\rho / h$ where $\rho$ is the resistivity and $h$ is the film thickness. For instance, organic photovoltaics require $10 \Omega$ per square sheet resistance which is achieved using $1 \mu \mathrm{m}$-thick films of highly conductive metals, like $\mathrm{Au}, \mathrm{Cu}$ and $\mathrm{Ag}$. There has been great progress in lowering the sheet resistance of films applied to substrates, and an excellent result was published recently, using a procedure based on graphite exfoliation under high shear rate. This was done in the presence of Na-carboxymethylcellulose at concentrations as high as $100 \mathrm{~g} / \mathrm{L}^{-1}$, and the authors achieved $<2 \Omega$ per square sheet resistance, which is already in the range required for printed RFID antennas.

The resistance of ERG coatings varies within a broad range, depending on the composition of the dispersion used, thickness and finishing procedure. The lowest value achieved is $0.6 \mathrm{ohms}$ per square, which is suitable for many macroscopic applications.

For instance, any square coating with this resistance dissipates only $1.3 \mathrm{~W}$ under a 15 A current. If the voltage fed to the circuit is $120 \mathrm{~V}$, the power dissipated in the coating square is less than $0.1 \%$.

In many applications, the conductive materials used should also comply with safety and environmental requirements, especially when the coated products are designed for a single use, e.g. in medical applications. Materials printed or coated with ERG do not pose any difficulties concerning final destination, since they can be composted or treated by various thermal processes (e.g. gasification, pyrolysis), leaving behind only carbon rich residues which are environmentally valuable. The graphite used in this work was of mineral origin, but useful conductive carbon particles are currently being produced from waste and they are showing promising results in conductive rubber. ${ }^{48}$ Each system has its pros and cons, considering the specifications set for specific applications. The procedures used in this laboratory to make conductive ink and paint are based on a mild, low-shearing procedure for partial exfoliation of graphite, forming high-aspect ratio nanographite sheets. Energetic procedures like sonication or milling are avoided, to prevent breaking of the graphite particles. High dispersant concentrations are also avoided, allowing for maximal contact among the individual graphene lamellae in the reassembled graphite.

ERG and even graphite are no match for the electrical conductivity of silver, but they have an advantage: they do not undergo significant oxidation under air. This is a problem for silver, in which a poorly conductive oxide is formed spontaneously under air, increasing the contact resistance among the silver particles and decreasing the shelf-life of silver-based conductive inks.

\section{The multiple roles of cellulose: exfoliating agent, adhesive, plasticizer}

The ability of aqueous cellulose solutions to wet and exfoliate graphite, while keeping the thin lamellae stably dispersed in an aqueous medium, is explained by the amphiphilic character of the individual cellulose chains.

ERG is made directly from the aqueous dispersion, avoiding the drying and powder handling steps which are required to make many current graphene products and compromise the quality of industrial products. ${ }^{57}$

Other properties of the extended thin ERG films described in the present work also derive from the properties of cellulose. ERG is resistant to water but it 
may swell, depending on the cellulose content. The moist films are plastic but they become rigid when dry. This is explained by the plasticizing role of cellulose chains interspersed with nanographite and graphene lamellae. The outcome is lamellae mobility, which allows their alignment along the film surface, exposing the basal plane to external electrical contacts. Low amounts of cellulose are required for coating cohesion and adhesion, as seen in the micrographs.

Moreover, cellulose chains are flat and they are likely adsorbed (within the dispersion) with a predominantly train conformation that leaves the flat graphitic surfaces largely unoccupied and suitable for electronic conduction.

The uniqueness of the contribution made by cellulose to ERG appears more clearly when we compare its performance to other widely used dispersants, for instance carboxymethylcellulose (CMC), a closely related modified cellulose. Graphite disperses well in aqueous solutions of CMC and the resulting dispersions can be used to paint paper, producing uniform coatings with low electrical resistance. However, the thin coating layers do not adhere strongly to flexible substrates: they do not withstand mechanical finishing procedures and they peel off the flexed substrates. This is a serious limitation in wearable circuitry. Moreover, CMC-based graphite coatings are easily re-dispersed in water and do not resist exposure to high humidity.

Exfoliating graphite and using it directly in its dispersions follows examples from the chemical industry, where highly unstable, sensitive or dangerous compounds, like phosgene, carbon disulphide, and sulphur trioxide as well as nickel and iron carbonyls seldom leave the plants to go anywhere. Using these chemicals in situ avoids huge handling, safety and environmental problems. In the present case, this approach combines top-down graphite exfoliation with its bottom-up reassembly as macroscopic continuous layers whose mechanical and handling properties differ pronouncedly from those of graphite powders or monoliths. The same approach can probably be used with other 2D materials to facilitate their use in various applications.

This is also in line with the work of many groups who have already opted for making graphene in situ, e.g. on copper foils. ${ }^{58}$

\section{Experimental}

\section{Reagents}

Different graphite types prepared by various procedures and with various particle dimensions were used in this laboratory, but the original results described in the present paper were obtained using graphite Grafine ${ }^{\circledR} 95100$ (Nacional de Grafite, São Paulo-SP, 96.4\% C, 3.5\% ash, mesh size $\geq 325$ ). Cellulose was used as the microcrystalline product Microcel@-101 (Blanver, São Paulo-SP, $50 \mu \mathrm{m}$ average particle size, $0.26-0.31 \mathrm{~g} \mathrm{~cm}^{-3}$ density), and the solution of $50 \% \mathrm{NaOH}$ in water was acquired from Synth (Diadema-SP).

\section{Sample preparation and handling procedures}

Graphite dispersions. Cellulose powder was slowly added to $5 \mathrm{~L}$ of a cold $\left(0-5{ }^{\circ} \mathrm{C}\right) \mathrm{NaOH}$ solution in a polyethylene beaker under gentle stirring using a Fisatom 713D high torque overhead stirrer at $210 \mathrm{rpm} \mathrm{min}^{-1}$ (the stirrer can 
handle up to $10 \mathrm{~L}$ liquid under $200 \mathrm{rpm}$ ) to avoid cellulose clumping. The resulting dispersion was kept in a refrigerator overnight, and then stirred and examined to ensure that the cellulose was fully dissolved. Cold water was added as required to achieve the desired cellulose concentration. The required amount of graphite was then slowly added to allow for intimate contact between the hydrophobic graphite powder and the cellulose solution. The powder was quickly wetted and sank within the solution, where it was allowed to exfoliate under gentle stirring or just stored at a low temperature. The same procedure was used for a broad range of concentrations: graphite, from 2 to $20 \%$ weight; cellulose, 0.5 to $4 \%$ weight; and $\mathrm{NaOH}, 3$ to $7 \%$ weight. Sonication and other energetic procedures were avoided to minimize cellulose chain and graphite lamellae breakdown. The dispersion viscosity was measured to verify the degree of exfoliation and the stability of the dispersions.

Substrate coating. Different coating techniques were used, but painting with a roll or brush was used most often in the laboratory, as follows: the desired amount of graphite/cellulose dispersion was weighed and spread over the substrate (paper, wood, cloth or other), using a polyester foam roll on smooth substrates or a polyester brush on rougher ones, distributing the dispersion evenly. It is important to avoid the formation of liquid pools on the substrate as well as any liquid runoff. Depending on the desired ERG coating weight per substrate unit area and the dispersion concentration, it may be necessary to do successive applications, allowing the coatings to dry in between them. Other procedures that were successfully used with different substrates are: spraying, spreading with a blade, silk-screening and lithography, using a mask. The quality of the substrate coating was monitored during the process by measuring the sheet resistance at various points to check the uniformity of the coating layer and also the extent of drying.

Finishing. Various procedures can be used, depending on the target sheet resistance, aesthetic and other requirements as well as the limitations posed by the substrate. Calendering produces the best results for paper and it was done using an MH Equipamentos (Guarulhos SP) MH300C model, while sanding and polishing are suitable for finishing ERG coatings on wood.

\section{Equipment and measurement procedures}

Scanning electron microscopy (SEM). Scanning electron microscopy (SEM) was performed on JSM-6360-LV thermionic equipment which uses a Noran system SIX/Thermo Electron Corporation with $\mathrm{Si}(\mathrm{Li})$ detector for energy dispersive spectroscopy (EDS). A Quanta-FEG/FEI with an Oxford $50 \mathrm{~mm}^{2}$ SSD detector was also used.

Raman spectroscopy. Raman spectroscopy was performed on a Confocal Horiba T64000 system using a $532 \mathrm{~nm}$ laser with nominal power of $7.5 \mathrm{~mW}$. Each spectrum was collected for $30 \mathrm{~s}$, and the material was observed for any visual damage.

Contact angle. Contact angle measurements were carried out on a Kruss Easy Drop apparatus, under ambient conditions. Water droplets were applied using a measuring syringe while the sample rested on a flat substrate. Pictures were taken using a CCD camera, and the contact angles were measured using the 
instrument software which found the drop and the substrate profile by detecting the respective contrast gradients.

Electrical measurements. Electrical measurements were made using Minipa (São Paulo) ET-2231A and Fluke 115 multi-meters. For routine sheet resistance monitoring, a contact cell was built from an acrylic block holding two parallel copper sheets. Square and contact resistance were measured by reading the resistance between successive points separated by $1 \mathrm{~cm}$ along a $1 \mathrm{~cm}$ wide conductive strip. The resistance was plotted as a function of distance. The slope gives the resistance per square while the intercept gives the contact resistance.

Atomic force microscopy (AFM). Samples for AFM measurements were cut into small square pieces (1 cm side length) and imaged in non-contact and contact modes. Topography maps were recorded on a Park NX10 microscope (Park Systems, Suwon - Korea) equipped with SmartScan software version 1.0.RTM11a. The electric force (EFM) measurements were conducted using a platinum coated n-type silicon probe (NSC14/Cr-Au, MikroMasch - Bulgaria) with a nominal resonance frequency of $160 \mathrm{kHz}$ and $5 \mathrm{~N} \mathrm{~m}^{-1}$ force constant. Lateral force microscopy (LFM) was done using a short aluminum backside-coated cantilever (PPP-CONTSCR, Nanosensors - Switzerland) with a nominal resonance frequency of $25 \mathrm{kHz}$ and $0.2 \mathrm{~N} \mathrm{~m}^{-1}$ force constant, and sample images were obtained at $90^{\circ}$ relative to the direction of the long axis of the cantilever. Conductive-probe AFM (CP-AFM) images were obtained using a solid platinum probe supported on a ceramic chip with a conductive gold bonding pad (25PT300B, Rock Mountain Nanotechnology - USA) with nominal resonance frequency of $100 \mathrm{kHz}$ and $250 \mathrm{~N}$ $\mathrm{m}^{-1}$ force constant. All measurements were made under ambient conditions at room temperature of $21 \pm 5{ }^{\circ} \mathrm{C}$ and relative humidity of $40 \pm 10 \%$. Images were treated using Park XEI software version 4.3.4Build22.RTM1. Each sample was imaged in three or more different regions.

\section{Conclusions}

Graphite dispersed in aqueous cellulose solutions is a bountiful source of new high-performance functional materials with various applications. This is largely due to the properties of cellulose which enable it to exfoliate graphite in aqueous solutions but also to perform as a plasticizing adhesive that allows the thin exfoliated lamellae to reassemble when they are applied on many different surfaces, forming extensive coating layers. Microscopy shows interesting features of the coatings which indicate the structural basis of their various macroscopic properties and applications. Coated paper and cloth can be bent to $<1 \mathrm{~cm}$ curvature radii and the eventual damage is easily repaired.

Changing the coating composition and surface finishing procedure produces a broad range of electrical properties, with surface resistance as low as $0.6 \mathrm{ohm}$ per square, close to the limit obtained for thick graphite layers and well below the value for single graphene layers. This is adequate for many applications of conductive materials in everyday use, in circuits integrated on clothes and in components of instruments and IT devices, machinery, and car and housing parts. Other applications involve the making of electrodes for electrochemical cells, supercapacitors, energy scavenging based on hygroelectricity, and electrodeposition on paper, wood and ceramic substrates. 
The fabrication processes offer many advantages: the raw materials are widely available commodities, and the coating fabrication and application are done using common procedures in the coating and paint industry. The only environmental risk in the making of these materials is the use of sodium hydroxide, which is commonly used in industry, e.g. to clean equipment. This chemical is neutralized by atmospheric $\mathrm{CO}_{2}$.

The exfoliated and reassembled graphite (ERG) shows other interesting features derived from the well-known properties of graphite: resistance to fire propagation, low friction, and high absorbance and emittance of light.

The singularities of ERG coatings derive from a number of unique properties of cellulose that are associated with its amphiphilic character: it is not soluble in common liquids, with very few exceptions. Dissolved cellulose adsorbs on many particulate solids, and the hydrophobic ring surface of its flat chain easily joins to graphene surfaces, producing spontaneous exfoliation of graphite under mild conditions. For these reasons, it is an excellent adhesive for exfoliated graphite thin lamellae, especially on cellulose surfaces.

Cellulose is plasticized by water and it can thus perform the role of a plasticizer within ERG. This endows the coatings with the ability to self-repair under high relative air humidity or when wet. For the same reason, successive coating layers show good mutual adhesion, and coating damage is quickly repaired by painting over it. This is further used in making circuit connections using graphite/cellulose as a conductive adhesive.

The approach used throughout this work circumvents some problems involved in making quality graphene (or even nanographite), and in storing, dispersing and applying it. Graphene is a high surface area non-polar substance that reaggregates easily, due to the well-known and irrevocable intermolecular van der Waals forces. Intensive efforts to produce high-quality graphene have been largely successful, and this is no longer the main difficulty responsible for its still low presence in multiple industrial applications where it is much needed.

\section{Conflicts of interest}

FG is one of the inventors listed in patent applications PCT/BR2016/050169, WO2017/063062A1, EP3363530 and US20180304210 and a partner in the GG \& FG company which is currently funded by FAPESP (São Paulo State Research Foundation) to develop the materials and applications described in this paper.

\section{Acknowledgements}

This work is supported by the Brazilian agencies MCTIC/CNPq (465452/2014-0), FAPESP (2014/50906-9) and Coordenação de Aperfeiçoamento de Pessoal de Nível Superior - Brazil (CAPES) - Finance Code 001 through INCT/INOMAT (National Institute for Complex Functional Materials). BCB thanks FAPESP (2016/02386-1), LPS thanks CAPES (88887.284776/2018-00) for a postdoctoral fellowship and FAPESP (2019/04565-9) for a research fellowship. Raman spectroscopy equipment was acquired by LMEOA (Institute of Physics of the University 
of Campinas) through grants EMU/FAPESP 2009/54066-7 and MCT/FINEP/CTInfra 02/2010. The authors thank LNNano (CNPEM, Campinas) for technical assistance and access to selected equipment. The authors thank Ramon H. Z. dos Santos for preparing samples and technical assistance with X-ray tomography measurements. This work is currently also supported by FAPESP PIPE project 2018/00834-2.

\section{References}

1 C. N. R. Rao, A. K. Sood, K. S. Subrahmanyam and A. Govindaraj, Angew. Chem., Int. Ed., 2009, 48, 7752-7777.

2 A. C. Ferrari, F. Bonaccorso, V. Fal'ko, K. S. Novoselov, S. Roche, P. Bøggild, S. Borini, F. H. L. Koppens, V. Palermo, N. Pugno, J. A. Garrido, R. Sordan, A. Bianco, L. Ballerini, M. Prato, E. Lidorikis, J. Kivioja, C. Marinelli, T. Ryhänen, A. Morpurgo, J. N. Coleman, V. Nicolosi, L. Colombo, A. Fert, M. Garcia-Hernandez, A. Bachtold, G. F. Schneider, F. Guinea, C. Dekker, M. Barbone, Z. Sun, C. Galiotis, A. N. Grigorenko, G. Konstantatos, A. Kis, M. Katsnelson, L. Vandersypen, A. Loiseau, V. Morandi, D. Neumaier, E. Treossi, V. Pellegrini, M. Polini, A. Tredicucci, G. M. Williams, B. Hee Hong, J.-H. Ahn, J. Min Kim, H. Zirath, B. J. van Wees, H. van der Zant, L. Occhipinti, A. Di Matteo, I. A. Kinloch, T. Seyller, E. Quesnel, X. Feng, K. Teo, N. Rupesinghe, P. Hakonen, S. R. T. Neil, Q. Tannock, T. Löfwander and J. Kinaret, Nanoscale, 2015, 7, 4598-4810.

3 Y. Zhu, H. Ji, H.-M. Cheng and R. S. Ruoff, Natl. Sci. Rev., 2018, 5, 90-101.

4 X. Li and L. Zhi, Chem. Soc. Rev., 2018, 47, 3189-3216.

5 H. Sun, J. Zhu, D. Baumann, L. Peng, Y. Xu, I. Shakir, Y. Huang and X. Duan, Nat. Rev. Mater., 2019, 4, 45-60.

6 A. Nag, A. Mitra and S. C. Mukhopadhyay, Sens. Actuators, A, 2018, 270, 177194.

7 G. Hu, J. Kang, L. W. T. Ng, X. Zhu, R. C. T. Howe, C. G. Jones, M. C. Hersam and T. Hasan, Chem. Soc. Rev., 2018, 47, 3265-3300.

8 D. Li, W.-Y. Lai, Y.-Z. Zhang and W. Huang, Adv. Mater., 2018, 30, 1704738.

9 X. Shi, S. Pei, F. Zhou, W. Ren, H.-M. Cheng, Z.-S. Wu and X. Bao, Energy Environ. Sci., 2019, 12, 1534-1541.

$10 \mathrm{X} . \mathrm{Xu}$ and Y.-L. Hsieh, Nanoscale, 2019, 11, 11719-11729.

11 K. R. Paton, E. Varrla, C. Backes, R. J. Smith, U. Khan, A. O’Neill, C. Boland, M. Lotya, O. M. Istrate, P. King, T. Higgins, S. Barwich, P. May, P. Puczkarski, I. Ahmed, M. Moebius, H. Pettersson, E. Long, J. Coelho, S. E. O'Brien, E. K. McGuire, B. M. Sanchez, G. S. Duesberg, N. McEvoy, T. J. Pennycook, C. Downing, A. Crossley, V. Nicolosi and J. N. Coleman, Nat. Mater., 2014, 13, 624.

12 G. Rao, X. Wang, Y. Wang, P. Wangyang, C. Yan, J. Chu, L. Xue, C. Gong, J. Huang, J. Xiong and Y. Li, InfoMat, 2019, 1, 272-288.

13 M. K. Jana and C. N. R. Rao, Philos. Trans. R. Soc., A, 2016, 374, 20150318.

14 Z. Yao, C. L. Kane and C. Dekker, Phys. Rev. Lett., 2000, 84, 2941-2944.

15 Y. Chen, X. Zhang, E. Liu, C. He, C. Shi, J. Li, P. Nash and N. Zhao, Sci. Rep., 2016, 6, 19363.

16 K. Chu, X. Wang, F. Wang, Y. Li, D. Huang, H. Liu, W. Ma, F. Liu and H. Zhang, Carbon, 2018, 127, 102-112. 
17 Y. Kim, J. Lee, M. S. Yeom, J. W. Shin, H. Kim, Y. Cui, J. W. Kysar, J. Hone, Y. Jung, S. Jeon and S. M. Han, Nat. Commun., 2013, 4, 2114.

18 D.-B. Xiong, M. Cao, Q. Guo, Z. Tan, G. Fan, Z. Li and D. Zhang, ACS Nano, 2015, 9, 6934-6943.

19 K. Zhao, T. Zhang, A. Ren, Y. Yang, P. Xiao, Z. Ge, Y. Ma and Y. Chen, Carbon, 2019, 141, 198-208.

20 E. S. Ferreira, D. S. da Silva, T. A. L. Burgo, B. C. Batista and F. Galembeck, Nanoscale, 2017, 9, 10219-10226.

21 J.-H. Ding, H.-R. Zhao and H.-B. Yu, Sci. Rep., 2018, 8, 5567.

22 J. Kim, S. Kwon, D.-H. Cho, B. Kang, H. Kwon, Y. Kim, S. O. Park, G. Y. Jung, E. Shin, W.-G. Kim, H. Lee, G. H. Ryu, M. Choi, T. H. Kim, J. Oh, S. Park, S. K. Kwak, S. W. Yoon, D. Byun, Z. Lee and C. Lee, Nat. Commun., 2015, 6, 8294.

23 V. León, J. M. González-Domínguez, J. L. G. Fierro, M. Prato and E. Vázquez, Nanoscale, 2016, 8, 14548-14555.

24 E. S. Ferreira, E. M. Lanzoni, C. A. R. Costa, C. Deneke, J. S. Bernardes and F. Galembeck, ACS Appl. Mater. Interfaces, 2015, 7, 18750-18758.

25 A. C. Ferrari, J. C. Meyer, V. Scardaci, C. Casiraghi, M. Lazzeri, F. Mauri, S. Piscanec, D. Jiang, K. S. Novoselov, S. Roth and A. K. Geim, Phys. Rev. Lett., 2006, 97, 187401.

26 W. Yang and C. Wang, J. Mater. Chem. C, 2016, 4, 7193-7207.

27 C. Liu, F. Li, L.-P. Ma and H.-M. Cheng, Adv. Mater., 2010, 22, E28-E62.

28 Y. Huang, J. Liang and Y. Chen, Small, 2012, 8, 1805-1834.

29 J. P. F. Bertacchi, Masters thesis, University of Campinas, 2019.

30 X. Liu, J. Wang and G. Yang, ACS Appl. Mater. Interfaces, 2018, 10, 20688-20695.

31 H. Wang, J. Deng, C. Xu, Y. Chen, F. Xu, J. Wang and Y. Wang, Energy Storage Materials, 2017, 7, 216-221.

32 K.-N. Kang, I.-H. Kim, A. Ramadoss, S.-I. Kim, J.-C. Yoon and J.-H. Jang, Phys. Chem. Chem. Phys., 2018, 20, 719-727.

33 P. He, J. Cao, H. Ding, C. Liu, J. Neilson, Z. Li, I. A. Kinloch and B. Derby, ACS Appl. Mater. Interfaces, 2019, 11, 32225-32234.

34 Z. Liu, F. Teng, C. Yuan, Z. Ul Abideen, W. Gu and Z. Liu, Energy Technol., 2019, 7, 1900314.

35 Y. Zhou, X. Wang, L. Acauan, E. Kalfon-Cohen, X. Ni, Y. Stein, K. K. Gleason and B. L. Wardle, Adv. Mater., 2019, 31, 1901916.

36 L. Manjakkal, W. T. Navaraj, C. G. Núñez and R. Dahiya, Adv. Sci., 2019, 6, 1802251.

37 T. R. D. Ducati, L. H. Simões and F. Galembeck, Langmuir, 2010, 26, 1376313766.

38 R. F. Gouveia and F. Galembeck, J. Am. Chem. Soc., 2009, 131, 11381-11386.

39 Y. Huang, H. Cheng, C. Yang, P. Zhang, Q. Liao, H. Yao, G. Shi and L. Qu, Nat. Commun., 2018, 9, 4166.

40 T. A. L. Burgo and F. Galembeck, J. Braz. Chem. Soc., 2016, 27, 229-238.

41 G. Xue, Y. Xu, T. Ding, J. Li, J. Yin, W. Fei, Y. Cao, J. Yu, L. Yuan, L. Gong, J. Chen, S. Deng, J. Zhou and W. Guo, Nat. Nanotechnol., 2017, 12, 317-321.

42 F. Zhao, Y. Liang, H. Cheng, L. Jiang and L. Qu, Energy Environ. Sci., 2016, 9, 912-916.

43 F. Zhao, H. Cheng, Z. Zhang, L. Jiang and L. Qu, Adv. Mater., 2015, 27, 43514357. 
44 J. Yin, X. Li, J. Yu, Z. Zhang, J. Zhou and W. Guo, Nat. Nanotechnol., 2014, 9, 378.

45 R. F. Gouveia, J. S. Bernardes, T. R. D. Ducati and F. Galembeck, Anal. Chem., 2012, 84, 10191-10198.

46 T. A. L. Burgo and F. Galembeck, Colloids and Interface Science Communications, 2015, 7, 7-11.

47 T. A. L. Burgo and F. Galembeck, Chemical Electrostatics, Springer, Cham, 2017.

48 K. Kampioti, C. F. Matos, F. Galembeck, C. Jaillet, A. Derré, A. J. G. Zarbin and A. Pénicaud, ACS Omega, 2018, 3, 1367-1373.

49 X.-Y. Fang, X.-X. Yu, H.-M. Zheng, H.-B. Jin, L. Wang and M.-S. Cao, Phys. Lett. A, 2015, 379, 2245-2251.

50 P. G. Karagiannidis, S. A. Hodge, L. Lombardi, F. Tomarchio, N. Decorde, S. Milana, I. Goykhman, Y. Su, S. V Mesite, D. N. Johnstone, R. K. Leary, P. A. Midgley, N. M. Pugno, F. Torrisi and A. C. Ferrari, ACS Nano, 2017, 11, 2742-2755.

51 R. V. K. Rao, K. V. Abhinav and P. S. Karthik, RSC Adv., 2015, 5, 77760-77790.

52 L. Hu, M. Pasta, F. La Mantia, L. Cui, S. Jeong, H. D. Deshazer, J. W. Choi, S. M. Han and Y. Cui, Nano Lett., 2010, 10, 708-714.

53 H. Zhang, Y. Qiao and Z. Lu, ACS Appl. Mater. Interfaces, 2016, 8, 32317-32323.

54 Y. Jiang, X. Ling, Z. Jiao, L. Li, Q. Ma, M. Wu, Y. Chu and B. Zhao, Electrochim. Acta, 2015, 153, 246-253.

55 D. J. Finn, M. Lotya and J. N. Coleman, ACS Appl. Mater. Interfaces, 2015, 7, 9254-9261.

56 B. Deng, P.-C. Hsu, G. Chen, B. N. Chandrashekar, L. Liao, Z. Ayitimuda, J. Wu, Y. Guo, L. Lin, Y. Zhou, M. Aisijiang, Q. Xie, Y. Cui, Z. Liu and H. Peng, Nano Lett., 2015, 15, 4206-4213.

57 A. Kovtun, E. Treossi, N. Mirotta, A. Scidà, A. Liscio, M. Christian, F. Valorosi, A. Boschi, R. J. Young, C. Galiotis, I. A. Kinloch, V. Morandi and V. Palermo, 2D Mater., 2019, 6, 25006.

58 J. Azpeitia, G. Otero-Irurueta, I. Palacio, J. I. Martinez, N. Ruiz del Árbol, G. Santoro, A. Gutiérrez, L. Aballe, M. Foerster, M. Kalbac, V. Vales, F. J. Mompeán, M. García-Hernández, J. A. Martín-Gago, C. Munuera and M. F. López, Carbon, 2017, 119, 535-543. 